\title{
LA DIMENSION SYSTÉMIQUE DU TRACÉ RÉGULATEUR : UNE STRUCTURE MUSICALE POUR L'ARCHITECTE
}

\author{
Author(s) / Auteur(s) : \\ Driss TAOUMI, diplômé en master d'architecture (École d'Architecture de Casablanca) \\ Doctorant en art de bâtir et urbanisme (Laboratoire Théorie des Systèmes en Architecture (tsa-lab)) \\ Faculté d'architecture, d'ingénierie architecturale, d'urbanisme (LOCI) \\ Université catholique de Louvain (UCLouvain) \\ driss.taoumi@student.uclouvain.be
}

\begin{abstract}
Résumé/Abstract :
"Tout trait doit être justifié !" quel étudiant architecte n'a pas été confronté à cette sentence ? Généralement, cette règle implicite est imposée sans être clairement explicitée. Ainsi, pour aller à l'encontre de cette injonction, un moyen clair est le tracé régulateur. Par définition, il est un tracé géométrique appliqué au dessin d'architecture ; permettant de géométriser ce dernier. Autrement dit, il sert à objectiver sa mise en forme, conformément à la science "dure" de la géométrie. Ce faisant, il habilite à justifier la construction des traits du dessin par une démonstration mathématique incisive, dissipant donc l'injonction "tout trait doit être justifié !". Simultanément à cette justification logique, le tracé régulateur donne lieu à des proportions, qui sont des rapports arithmétiques - et visuels - entre deux dimensions (1/2, 2/3, 3/4, nombre d'or...). Ils permettent alors une mise en relation des parties du dessin, dont le résultat est leur cohésion - ou musicalité - graphique. Par ailleurs dans la conception/dessin d'architecture en particulier, la proportion établit ces rapports entre les dimensions des traits, aussi bien qu'entre leurs positions. Ils en deviennent alors un système statique, soit une structure organique : si la dimension et/ou la position d'un trait change, alors les dimensions et positions des autres traits sont aussi altérées. Cette interrelation dimensionnelle et topologique constitue l'aspect systémique du tracé régulateur.
\end{abstract}

\section{Keywords / Mots-clés :}

architecture, tracé régulateur, tracé harmonique, proportions, nombre d'or, systémique, modulor, Corbusier

\section{ORIGINE ET DÉFINITION DE L'EXPRESSION "TRACÉ RÉGULATEUR"}

En apparence, l'expression "tracé régulateur" a été énoncée d'abord par le Corbusier, dans son livre iconique Vers une architecture (fig. 1). À première vue, elle semble lui avoir été inspirée par les écrits d'Auguste Choisy, notamment ceux d'Histoire de l'architecture (1899). Par cette élocution, il désigne un tracé géométrique permettant de "réguler" la mise en forme d'une architecture. D'une part, le terme "réguler" fait référence à la notion de règle, dans le sens où elle permet de dompter, de comprendre avec précision la mise en forme d'une conception. Sans doute, cette terminologie de régulation est

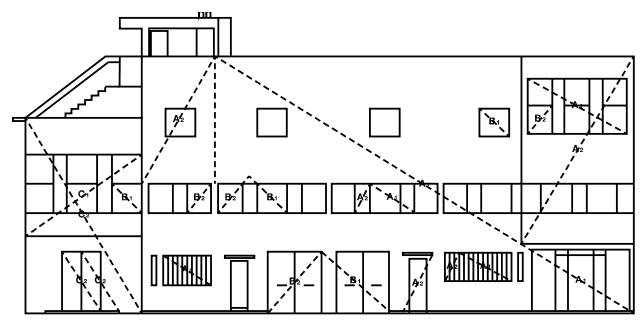

Figure 1 : Tracé régulateur de la villa "La Roche" d'après le Corbusier dans Vers une architecture (Driss TAOUMI 2019) propice, car effectivement, le tracé géométrique permet une maîtrise scientifique de cette mise en forme architecturale, puisqu'il habilite à la construire de manière exacte et mathématique. Ainsi, cette construction morphologique absolue en devient universellement intelligible, au même titre que la démonstration d'une équation arithmétique. D'autre part, "réguler" fait aussi référence à la règle dans son acception de rythme musical, de tempo structurant la symphonie. Ici encore, la terminologie de régulation est fondée, car les incidences géométriques du tracé régulateur produisent des proportions, et celles-ci permettent en effet d'accorder les rythmes graphiques d'une conception architecturale. Toutefois, le champ lexical de la règle étant associé à l'idée de punition, d'autres auteurs préfèrent l'appellation de "tracé harmonique" à celle de "tracé régulateur". 
Malgré tout, il faut noter que la sonorité de "tracé régulateur" est préférable à celle de "tracé harmonique", car cette dernière juxtapose maladroitement une syllabe vide avec un $h$ muet (traCÉ HARmonique), tandis que "tracé régulateur" combine élégamment syllabe vide et pleine (traCÉ RÉgulateur), d'où sa prononciation plus agréable, et donc sa démocratisation facilitée. Cela dit, il est un tracé géométrique appliqué à la mise en forme d'une architecture : il permet de la géométriser, d'abord pour une compréhensibilité universellement intelligible de sa construction morphologique absolue, et ensuite pour sa cohésion visuelle à travers ses proportions géométriques incidentes. Exemple de tracé régulateur

\section{LA PLACE DU TRACÉ RÉGULATEUR DANS LA DISCIPLINE DE LA GÉOMÉTRIE}

Étymologiquement, la géométrie ${ }^{1}$ est la mesure de la terre, et permet ainsi de mesurer les dimensions de celle-ci. Mais au lieu de mesurer chacune individuellement, on peut les déduire sans efforts et sans erreurs avec les théorèmes géométriques (fig. 2), si on connaît les valeurs avec lesquelles elles sont en relation, aussi bien que cette relation elle-même : ce procédé constitue le calcul. Il sert donc à déduire des angles, des longueurs, des surfaces, et des volumes. En somme, la géométrie est un outil de calcul logique et intellectuel, et cela qualifie son aspect utile ${ }^{2}$. D'autre part, elle est aussi utilisée comme moyen d'illustration, à cause de sa nature graphique et expérientielle : elle est souvent désignée de géométrie parfaite ou symphonique - voire sacrée (fig. 3). Ce caractère visuellement plaisant constitue son aspect sensuel ${ }^{3}$, et nous nous y intéresserons exclusivement dans cet article : la géométrie en tant qu'outil de mise en forme, appliquée comme tracé régulateur pour géométriser (absolutiser) la morphologie d'une conception architecturale. Entre autres, le tracé régulateur est un dessin ${ }^{4}$ géométrique traditionnel, car il emploie les outils de dessin conventionnels, soient-ils analogiques ou numériques :

- Outils de tracé (traces dans le sable, cordes, plume, stylo à bille, vecteurs des logiciels de dessin...);

- Outils d'angles et traits droits (corde à 13 nœuds, équerre, fonction verrouiller angles/traits droits des logiciels de dessin...);

- Outils de rotation (corde avec piquet, compas, fonction cercle des logiciels de $\operatorname{dessin} . .$.

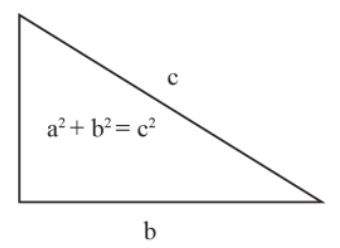

Figure 2 : Théorème de Pythagore, géométrie de calcul utile (Driss

TAOUMI 2019)

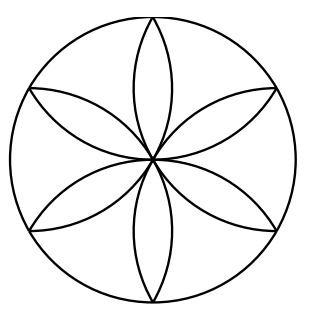

Figure 3 : Rosace à six pétales, tracé géométrique expérientiel et sensuel (Driss TAOUMI 2019)

\footnotetext{
1 "Géométrie" vient des mots grecs géo (terre) et métron (mesure), elle est donc la mesure de la terre.

2 Qualité d'outil, de ce qui sert à quelque chose. L'outil facilite les opérations exécutées par l'être humain, en réduisant l'utilisation de ressources (temps, effort, matières premières...), tout en augmentant la quantité et la qualité du résultat. En somme, il permet de faire plus, mieux, avec moins.

Exemple 1 : calculer avec des théorèmes est utile car cela permet de déduire des mesures, sans avoir à aller sur le terrain pour les mesurer individuellement (ce qui multiplie le risque d'erreur, le temps utilisé, et les personnes mobilisées). Le calcul a donc réduit le temps, l'effort, et les ressources employées, tout en augmentant la qualité et la quantité de mesures possibles. Exemple 2 : le théorème de Pythagore est utile, car l'angle droit qu'il définit facilite l'égalisation de surfaces (quadrillage) en minimisant les directions du tracé, réduisant ainsi les efforts, le temps, et les erreurs de tracé. En comparaison, les angles non droits compliquent le quadrillage, parce qu'ils augmentent les directions du tracé, multipliant donc les efforts, le temps, et les erreurs de tracé. D'où l'utilité du théorème de Pythagore, puisqu'il permet de faire plus, mieux, avec moins (figure 5).

3 Qualité de ce qui est relatif aux sens, de ce qui les stimule. Dans le cas du tracé régulateur, le sens sollicité est celui de la vue.

${ }^{4}$ En architecture, le dessin est une production graphique avec/dans laquelle l'architecte donne forme/trouve ses idées : de manière organique, il les produit intellectuellement et intuitivement, en les concrétisant à travers des traces sur une surface ou dans un volume, pour pouvoir les communiquer à lui-même ou à autrui. Ce dessin peut être réalisé sur le média conventionnel du papier, sur écran, à l'intérieur d'une réalité virtuelle ou dans un hologramme.
} 


\section{Tracé géométrique régulateur}

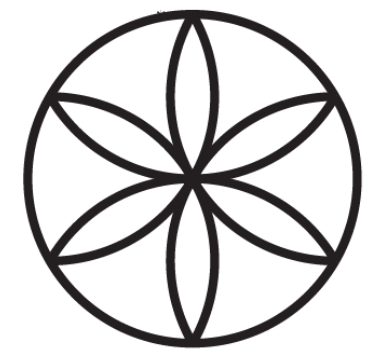

Figure 4 : Les aspects complémentaires du tracé régulateur (Driss TAOUMI 2019)

\section{Construction}

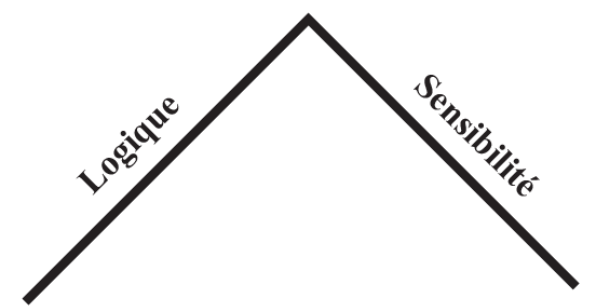

par blocs constructifs

Harmonisation par proportions

1) $P \bullet$ int (ou intersection)

Proportion de segments
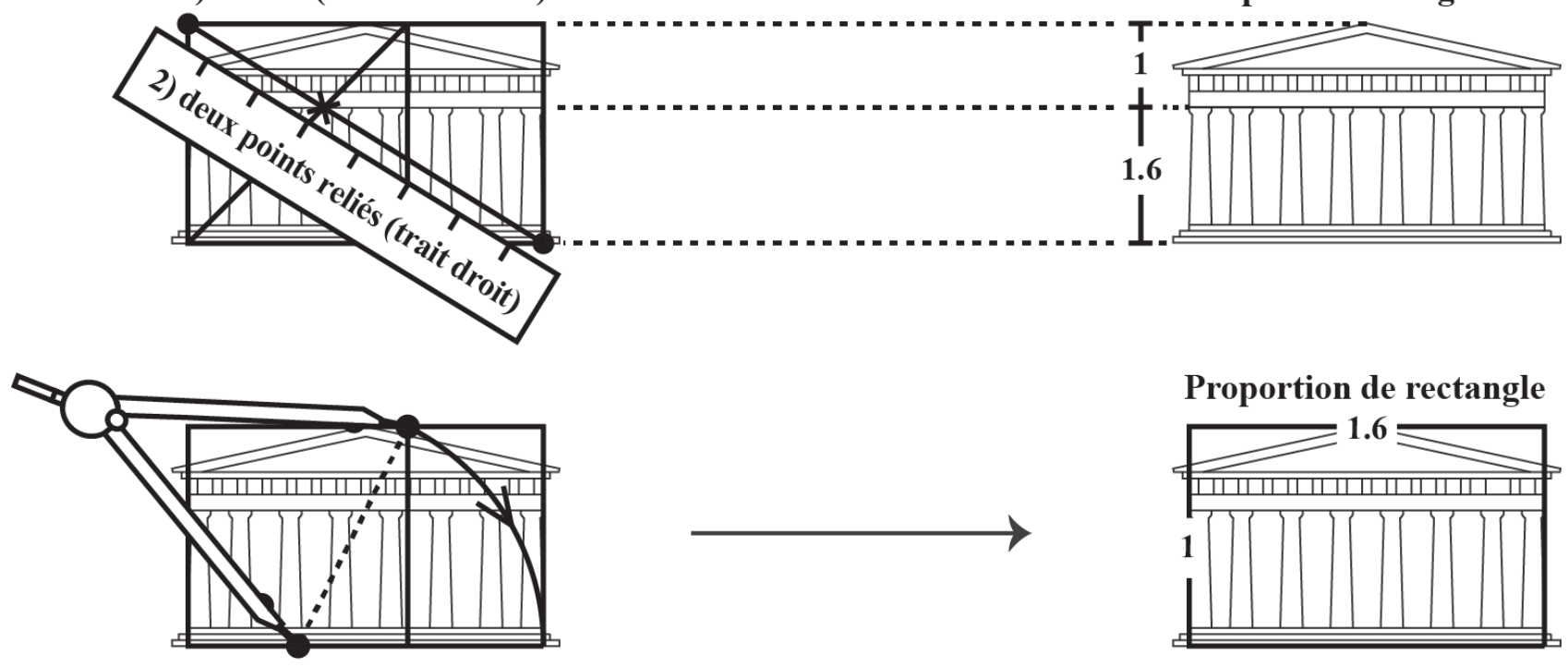

Proportion de rectangle

3) Point tournant autour d'un autre (trait courbe)
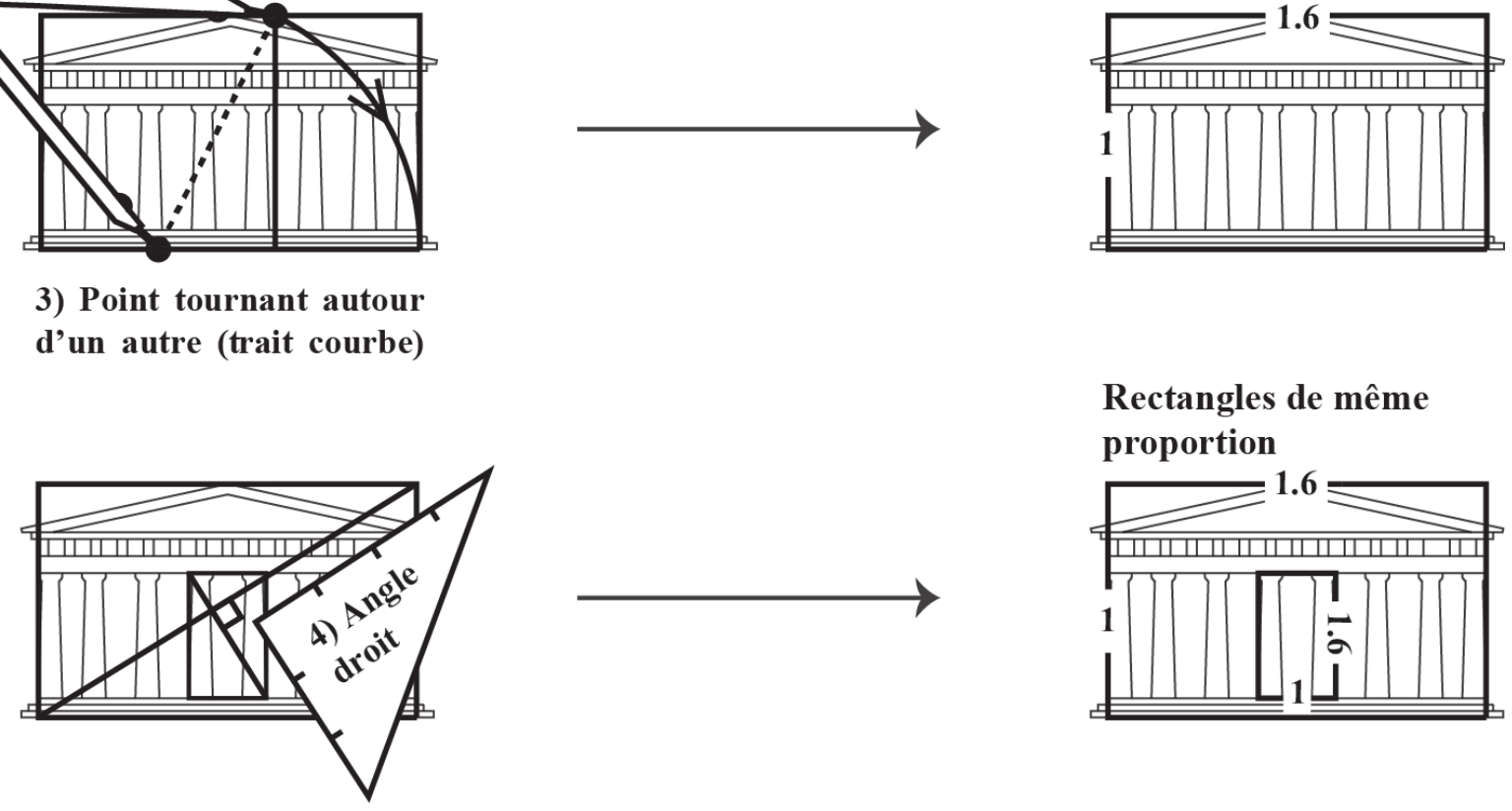

Rectangles de même proportion

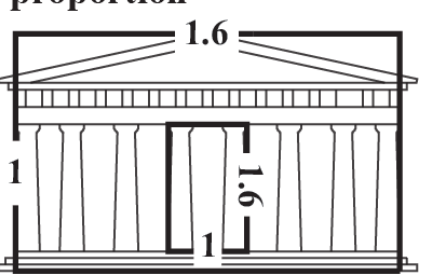




\section{Égalisation de surfaces à angles droits (2 directions)}

Le schéma suivant explique l'utilité de l'angle droit défini par le théorème de Pythagore (géométrie de calcul) : il est l'angle de travail le plus simple, car il minimise le nombre de directions produites.

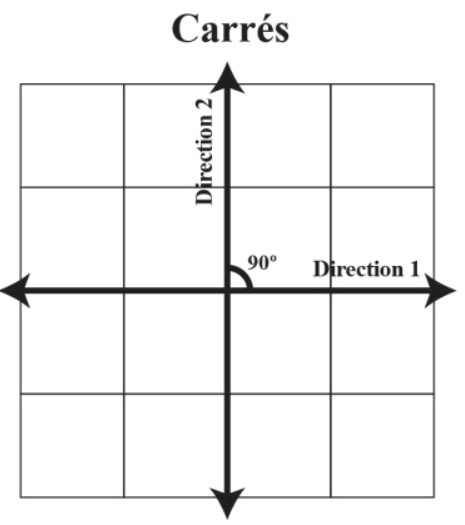

\section{Rectangles}

Figure 5 : Utilité de l'angle droit défini par le théorème de Pythagore. Les surfaces à angles droits sont plus utiles au quadrillage, car elles minimisent les efforts, le temps, et les erreurs de tracé, tout en augmentant la compréhensibilité et l'accessibilité de ce quadrillage. Ce faisant, il semble que les villes quadrillées à l'angle droit sont plus accessibles et compréhensibles, car il paraît qu'on s'y perd moins. (Driss TAOUMI 2019)

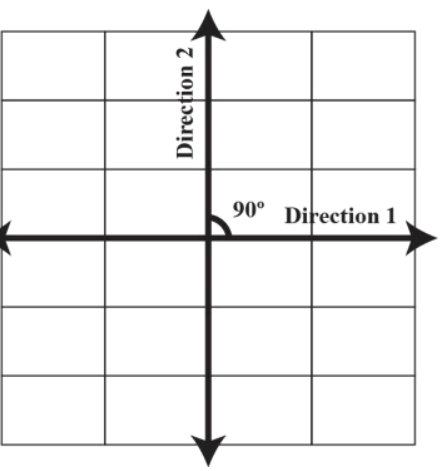

nombre de directions minimal: efforts, temps, et erreurs de tracé réduits, compréhensibilité et accessibilité augmentées

\section{Égalisation de surfaces à angles non droits (plus de 2 directions)}
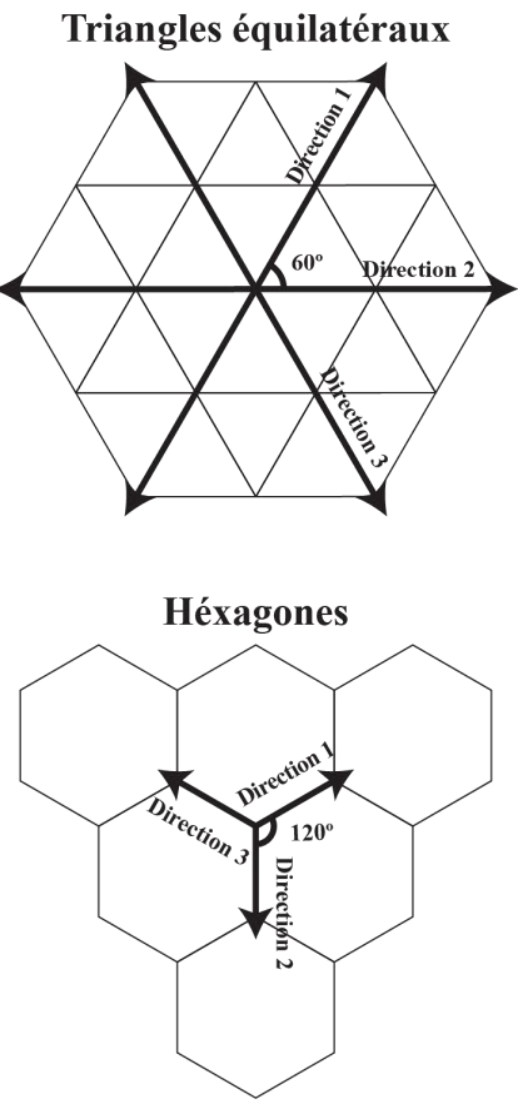

nombre de directions plus grand: efforts, temps, erreurs de tracé augmentés, compréhensibilité et accessibilité réduites

\section{HISTORIQUE DU TRACÉ RÉGULATEUR, ET SON INTÉRET AU XXI' SIECLE}

Cet historique est une synthèse personnelle de souvenirs de lectures, il a donc peu de valeur scientifique, et n'est présent dans cet article qu'à titre illustratif :

À travers l'histoire, l'être humain a employé le tracé régulateur pour géométriser le dessin d'architecture, ceci afin de permettre la maîtrise de sa construction morphologique absolue, aussi bien que la mise en relation mélodieuse de ses parties, satisfaisant ainsi l'instinct artistique et mathématique des architectes. Au départ avec l'être humain primitif des tribus disparates, il n'y a pas encore de tracé régulateur, mais seulement une sensibilité arithmétique, qu'il exprima en utilisant des modules simples et rationnels (Le Corbusier, Vers une architecture, 1923) (fig. 6). Il faut attendre les grandes civilisations Mésopotamie, Égypte antique, Grèce antique... - pour voir
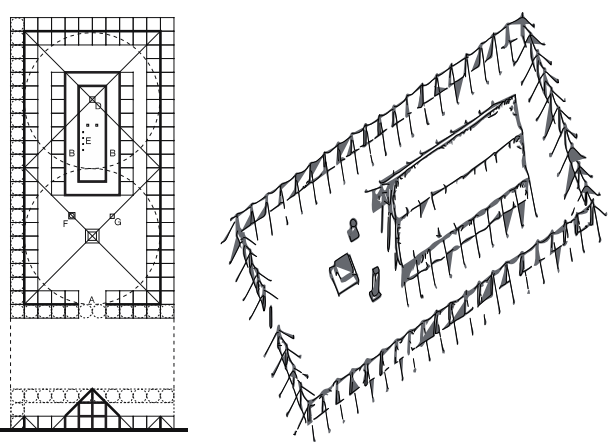

Figure 6 : Plan, coupe, et axonométrie d'un temple primitif. Son agencement est intégralement soumis à un module mathématique, le tout selon le Corbusier dans Vers une architecture (Driss TAOUMI 2019) naître le tracé géométrique régulateur et ses vérités métaphysiques irrationnelles (ex. : nombre d'or). On l'appliqua aux architectures importantes de l'époque (constructions polythéistes) : ziggurats, pyramides, 
temples, mausolées... Ensuite, le tracé régulateur est appliqué à l'architecture monothéiste : synagogues, églises, cathédrales, mosquées... Puis dans la renaissance humaniste occidentale, on veut effectivement se détacher de Dieu pour revenir à l'être humain. Il en est de même pour le tracé régulateur : au lieu de l'appliquer seulement aux constructions divines, on ose l'utiliser sur quelques bâtiments à clientèle humaine : palais des riches, écoles, bâtiments politiques... Plus tard, après la révolution industrielle et l'apparition des machines, le Corbusier proclame qu'une maison doit être faite avec autant de soin qu'un palais "une maison - un palais" (Le Corbusier, Vers une architecture, 1923), et veut donc appliquer le tracé régulateur à des constructions encore plus modestes. À sa malchance, le petit peuple pour qui il voulait construire n'était guère apte à se permettre de soins architecturaux. Ainsi contraint à ne pas pouvoir choisir son client, il applique le tracé régulateur dans des villas bourgeoises, mais surtout dans son logement de masse "l'Unité d'Habitation", projet dans lequel il a finalement pu loger les populations moyennes qu'il visait au départ.

Enfin, dans l'architecture du XXI ${ }^{\mathrm{e}}$ siècle réalisée à l'aide de l'outil numérique, la tridimensionnalité de la conception devient plus forte et renie le tracé régulateur, comme il est une géométrie plate en deux dimensions. D'ailleurs, il est vite oublié au profit d'une régulation 3D en apparence obscure, car calculée automatiquement par l'instrument digital indompté. Mais encore, il a aussi été oublié que la géométrie du tracé régulateur constitue la base du logiciel informatique 3D. Par conséquent, on constate que l'architecte est aujourd'hui aliéné de son projet par l'outil numérique (fig. 7) : il lui permet de projeter des édifices aux formes sophistiquées, mais s'il ignore la géométrie sousjacente avec laquelle le logiciel construit ces formes, comment l'architecte peut-il prétendre maîtriser leur construction virtuelle, encore moins leur construction dans le réel ? Fatidiquement, l'architecte aura beau se complaire dans ses édifices

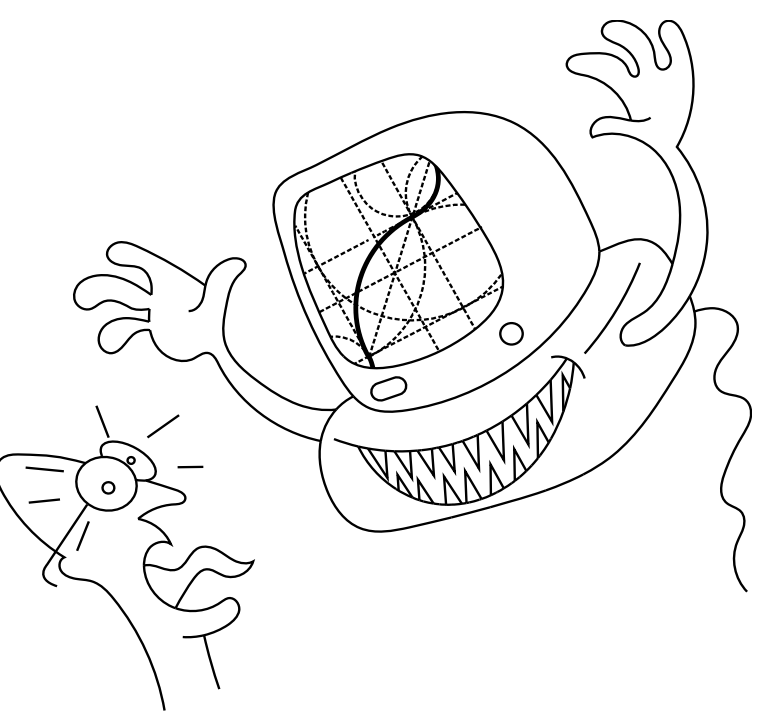

Fig. 7 : L'architecte aliéné de son projet par l'outil numérique : il a négligé la géométrie basale sur laquelle fonctionne celui-ci (Driss TAOUMI 2019) aux morphologies sculpturales, mais s'il ne maitrise pas la géométrie avec laquelle son programme les bâtit virtuellement, ce sera ce dernier qui aura été l'auteur de leur construction virtuelle, et l'ingénieur aura été l'auteur de leur construction réelle... Mais alors, quelle aura été la contribution de l'architecte dans ce procédé de fabrique ? Les apôtres de Frank Gehry contribuent en froissant une feuille qu'ils qualifient de "projet", et l'emberlificotent d'un discours vague qu'ils appellent "l'idée du concept". Cette tendance semble croître dans les écoles d'architecture d'influence française, il n'est donc pas étonnant que l'architecte perde peu à peu sa place de concepteur dans ce contexte. Et cette position sera de toute façon revendiquée par l'ordinateur intelligent, car si l'architecte le laisse maîtriser l'activité de conception à sa place, ce sera bientôt l'ordinateur qui maîtrisera l'architecte, et cela s'avère d'autant plus actuel avec l'émergence de l'intelligence artificielle.

Hélas, l'architecte du XXI ${ }^{\mathrm{e}}$ siècle veut hâtivement se précipiter vers l'avenir inconnu, sans vouloir prêter attention aux fondements qui lui ont légué son outil sophistiqué, afin de porter tant soit peu le flambeau de l'innovation: "petit succès prématuré ; la ruine est proche, on ne bâtit pas sur le sable." son affalement fatidique et inévitable ne nous étonnera pas, puisque son mouvement vers l'avant n'était pas suffisamment ancré au sol, étant donné sa négligence de la racine qui lui a bourgeonné son logiciel : le tracé géométrique régulateur. D'où l'intérêt d'un rappel renouvelé de cette géométrie basale, qui nous évitera l'effondrement fatidique de l'histoire répétée, au profit d'un pas lucide et assuré vers l'avant. Pour résumer l'intérêt du tracé régulateur dans la pratique architecturale contemporaine, il convient de citer cet adage célèbre, vu qu'il correspond aujourd'hui à la situation de l'architecte, et à celle de l'être humain actuel en règle générale : "Celui qui ne sait pas d'où il vient, ne peut savoir où il va, car il ne sait pas où il est. En ce sens, le passé est la rampe de lancement vers l'avenir." 


\section{L'aspect intellectuel du tracé régulateur : la constructibilité}

Le tracé régulateur permet de construire la morphologie d'une architecture. Cette construction logique emploie des blocs de constructions (fig. 8) : le point, le trait droit (deux points reliés), l'angle droit, le trait courbe (point qui tourne autour de l'autre). De manière raisonnée, l'architecte assemble sa conception avec ces briques abstraites, comme le maçon qui assemble son mur avec ses briques de terre. D'autre part, quand il trace géométriquement, son processus de dessin devient logique : clair, exact et maîtrisé. Autrement dit, il sait précisément d'où vient chaque point, chaque trait, chaque courbe. Désormais, il n'y a plus de hasard dans la mise en forme d'une morphologie architecturale, car elle est intégralement démontrable par un tracé mathématique absolu. De ce fait, les positions et les dimensions de chaque trait sont verrouillées avec ce tracé préalablement choisi par l'architecte ; l'intellect ${ }^{5}$ et la logique sont satisfaits. Enfin, la question "pourquoi ce trait ?" est dissipée. Grâce au tracé régulateur, l'anatomie formelle de l'architecture devient compréhensible par tout un chacun, parce qu'elle peut être soumise à une démonstration géométrique universellement intelligible, comme en témoigne le Corbusier : "Le tracé régulateur est le ce qu'il faut démontrer (CQFD) du mathématicien" (Le Corbusier, Vers une architecture, 1923)

\section{L'aspect sensuel du tracé régulateur : la mise en relation visuelle}

En plus d'être un outil de construction géométrique, le tracé régulateur permet aussi de corriger graphiquement la morphologie d'une architecture. Il s'agit d'accorder ses parties en y introduisant des liens visuels : les proportions (fig. 9) : l'unité, la moitié, le tiers, le quart, le cinquième, le nombre d'or/d'argent/de bronze/plastique, les racines carrées, les rectangles de même proportion... Avec ces liens arithmétiques, les parties d'un bâtiment forment un tout visuellement musical : elles vont bien ensemble. D'où le concept de "musicalité" que décrit Pythagore avec les sons harmonieux de son monocorde ${ }^{6}$, quand pincé aux proportions mathématiques précédentes. En effet, ces dernières satisfont le complémentaire de l'intellect : l'esprit ${ }^{7}$ et la sensibilité.
1) Le point $\bullet$

2) Deux points reliés (trait droit)

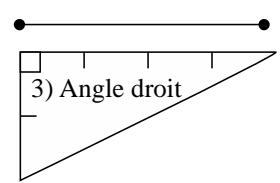

4) Point qui tourne autour de l'autre (trait courbe)

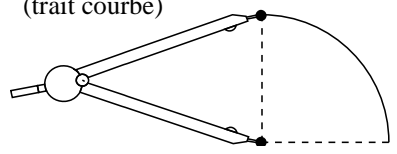

Fig. 8 : Les blocs constructifs du tracé régulateur (Driss TAOUMI 2019)

"Voici les tracés régulateurs qui ont servi à faire de très belles choses, et qui sont la raison pour laquelle ces choses sont si belles." (Le Corbusier, Vers une architecture, 1923). Ainsi dans cette citation, le Corbusier décrit l'aspect sensuel du tracé régulateur par le qualificatif "belle". Il y caractérise l'inclusivité graphique que les proportions permettent : elles unifient la mise en forme d'une "chose", et lui apportent alors cette dite "beauté". De plus, il est aussi l'auteur de la citation suivante, qui se veut parallèle à l'idée de sensualité : "Le tracé régulateur procure la satisfaction de l'esprit." (Le Corbusier, Vers une architecture, 1923). Ici, la spiritualité qu'il décrit n'est pas celle de l'ésotérisme, mais toujours ce sentiment de cohésion visuelle que produisent les proportions du tracé régulateur: elles font que les parties d'un dessin architectural ne soient plus disparates, et paraissent désormais venir d'une même famille de formes. Autrement dit, elles deviennent accordées dans leurs rythmes visuels, comme le caractérise Pythagore avec son monocorde et ses sons accordés. En somme, les proportions du tracé régulateur transforment les parties d'une conception en un système statique,

\footnotetext{
5 L'intellect est la faculté humaine d'abstraction, qui s'effectue dans le monde des idées et par la réflexion dans l'absolu. Elle permet une virtualisation mentale gouvernée exclusivement par la logique, et a pour caractéristique d'être sans aléas. L'intellect est souvent symbolisé par l'image du cerveau ou de la tête pensante.

6 Expliqué dans la figure 11

7 L'esprit est un sentiment issu de la cohésion organique d'un phénomène : la participation harmonisée de tous ses intervenants. Dans la morphologie d'une architecture, les intervenants sont les parties. Si on considère l'expression communément admise "le tout est supérieur à la somme des parties" l'esprit constitue exactement cette différence entre le tout et la somme des parties, si et seulement si elles sont mélodieusement accordées. Il s'avère être une nécessité métaphysique à l'être humain.
} 
où elles sont toutes en interrelation dimensionnelle et topologique. Cela dit, l'architecte choisit lui-même leurs modalités d'interrelation proportionnelle à l'aide de son goût artistique, de concert avec les solutions rationnelles qu'imposent la biologie et les contraintes de son architecture. "Les édifices, les uns sont muets ; les autres parlent ; et d'autres enfin chantent." (Paul Valéry, Eupalinos ou l'architecte, 1921). Dans cette citation, l'auteur décrit le même phénomène spirituel que le Corbusier énonce, et lui transpose une qualité de chant musical. Encore une fois, cela rejoint l'expérience du monocorde pythagoricien : les proportions sont le lien musical entre les sons auditifs, et s'avèrent aussi valable pour les sons visuels d'une architecture, selon ce qu'implique Valéry. Certainement, elles sont ici précisées avec l'utilisation du verbe "chanter" : l'écrivain remarque que certains bâtiments ne font que "parler" - leurs parties ne s'accordent pas et font du bruit visuel disparate (comme dans le jazz) - tandis que d'autres parlent harmonieusement - leurs sons visuels sont élégamment accordés par les proportions d'un tracé régulateur - ils "chantent". Cette transposition musicale est commune dans le sujet des proportions, car elles sont universellement responsables du phénomène mélodique, indépendamment de sa nature auditive ou visuelle. "Un bâtiment moderne doit tirer sa signification architecturale uniquement de la vigueur et de la conséquence de ses propres proportions organiques." (Walter Gropius, The new architecture and the Bauhaus, 1925). Encore et toujours, Walter Gropius apporte le même message que le Corbusier et Paul Valéry, il remplace seulement "beauté, esprit, et chant" par "signification architecturale".

\section{LE TRACÉ RÉGULATEUR INFLUENCE LA CONCEPTION, MAIS SERT SURTOUT A CONSTRUIRE ET A CORRIGER VISUELLEMENT UNE MORPHOLOGIE}

Pour faciliter l'explication, nous avons séparé ces deux aspects du tracé régulateur - aspect utile (construction) et aspect musical (correction visuelle) - qui sont en réalité inséparables : quand on réalise un tracé régulateur, on applique les deux simultanément.

En apparence, le tracé régulateur n'est pas un outil de conception. Cependant, comme il modifie les positions et les dimensions des éléments architectoniques, il influe donc sur la conception de ceux-ci, et peut par conséquent causer le renouvellement entier d'un projet. Nous ne maîtrisons pas cette piste, et elle mériterait une investigation qui lui est propre. Cela dit, les fonctions claires du tracé régulateur sont : construire le dessin d'une morphologie (utilité) et le corriger (musicalité).

1) Construire le dessin d'une morphologie : l'assembler avec un tracé géométrique préalablement choisi, pour la maîtriser logiquement et exactement dans tous ses détails, afin qu'elle constitue l'œuvre d'un professionnel plutôt que celui d'un amateur. Cette construction mathématique justifie les positions et les dimensions des traits du dessin, en leur permettant d'être universellement intelligibles et démontrables. Cela constitue l'aspect utile du tracé régulateur, qui est une justification brute. Elle satisfait l'intellect et la logique.

2) Corriger le dessin d'une morphologie : mettre en relation ses parties avec les proportions formées par les incidences géométriques, afin qu'elle devienne graphiquement mélodieuse. C'est l'ajout de la cohésion visuelle à la justification mathématique brute : l'esprit et la sensibilité sont satisfaits. D'ailleurs, le Corbusier

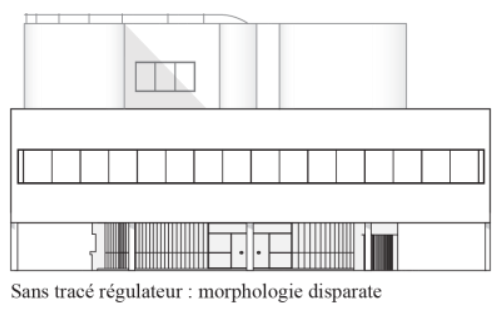

Sans tracé régulateur : morphologie disparate

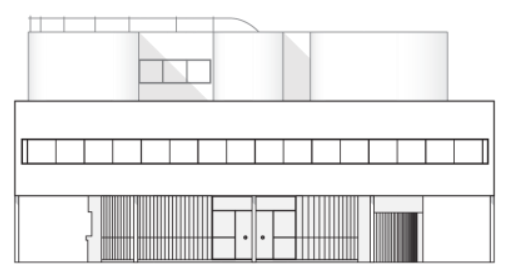

Avec tracé régulateur : morphologie cohésive

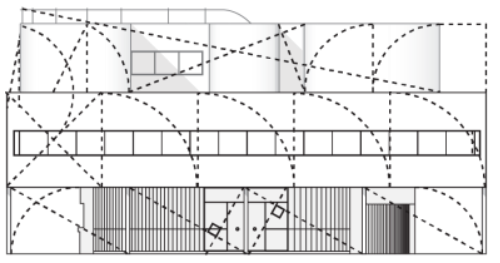
Intégralité organique du tracé régulateur : justification géométrique et mise en relation (correction) des parties morphologiques architecturales

Fig. 10 : Construction mathématique et correction visuelle par tracé régulateur (Driss TAOUMI 2019) déclare que les architectes mémorables corrigeaient aussi leurs productions graphiques à l'aide du tracé régulateur : "Le Grec, l'Égyptien, Michel-Ange ou Blondel employaient les tracés régulateurs pour la correction de leurs ouvrages." (Le Corbusier, Vers une architecture, 1923). Entre autres, le tracé régulateur permet une justification identique à celle de Microsoft Word : elle géométrise les extrémités de paragraphe, 
en leur faisant former des lignes droites géométriques. La différence avec le tracé régulateur est que les modalités de justification sont infiniment plus riches. En somme, plus nous cultiverons notre connaissance du tracé géométrique, plus il nous habilitera à dompter l'anatomie formelle de nos architectures, tout en les rendant fertiles à l'harmonie visuelle. Par ailleurs, si on contextualise le tracé régulateur dans l'élaboration d'un projet : Conception > Différentes itérations du projet > Tracé régulateur. Ici en apparence, il s'applique suivant cet ordre, mais il influence peut-être la conception en amont, car sa pratique préalable produit un guide inconscient de composition.

\section{TRACÉ RÉGULATEUR, DESSIN D'ARCHITECTURE, MUSIQUE ; QUELLE RELATION ?}

D'emblée, la durée différencie la musique du dessin d'architecture : l'appréciation d'une mélodie requiert une durée d'écoute (chanson de 3 minutes, ou symphonie de 30 minutes...) tandis que le dessin d'architecture ne demande qu'un instant de lecture (5 à 10 secondes). En somme, la musique est une symphonie dynamique temporelle, tandis que le dessin d'architecture est une symphonie statique spatiale. Par ailleurs, les sons de ce dernier sont ses éléments visuels (son tout, ses parties, ses portes, ses fenêtres, ses cages d'escaliers...), alors que le tout et les parties de la musique sont ses sons. De plus, un son tout seul ne peut être musical, mais plusieurs sons qui vont bien ensemble, et immédiatement nous percevons un début de musique : cet accord harmonieux est la proportion.

De plus, le même phénomène s'applique au dessin d'architecture : plusieurs sons visuels proportionnés, et immédiatement un phénomène plaisant apparaît. Sans doute, la cause en est la même que pour la musique : la proportion. Effectivement, dans les instruments à cordes, nous obtenons des sons mélodieux par proportionnement de ces cordes, connaissance qui a été apparemment découverte par Pythagore avec son expérience du monocorde ${ }^{8}$ (fig. 11). Par ailleurs, à la renaissance du XVè siècle, la redécouverte des proportions pythagoriciennes paraît contribuer à l'éclairement des esprits : elles

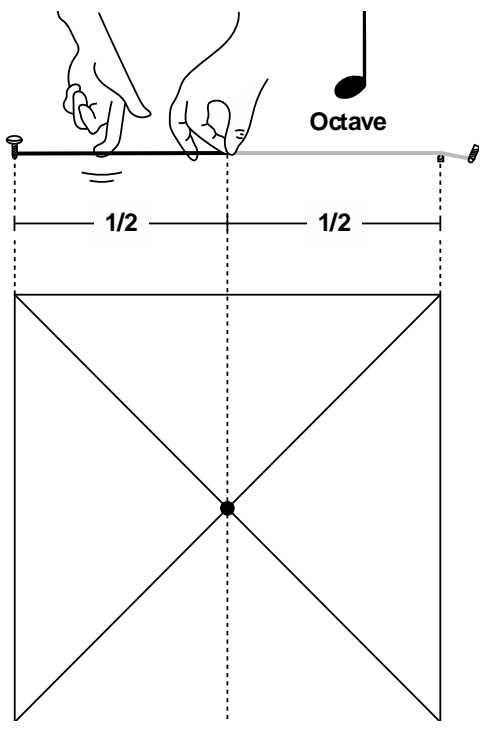

Figure 11 : Proportion de moitié, en tracé régulateur et dans le monocorde de Pythagore (Driss TAOUMI 2019) sensibilisent à la nature mathématique de l'univers et de la création (Galilée, L'essayeur, 1623) puisqu'elles sont constantes dans les dimensions sensitives de celle-ci. Ainsi, étant donné que nous - êtres humains - faisons partie de la création, les proportions musicales permettent donc la connaissance de soi, d'où leur qualité éclairante et révélatrice. Parallèlement entre dessin d'architecture et musique, nous obtenons des sons visuels musicaux par proportionnement des dimensions, et cela constitue la qualité même du tracé régulateur. Il faut noter que comme dans la musique, les gammes visuelles des proportions ne déterminent jamais la qualité de l'œuvre, car elles ne sont qu' un instrument visuel accordé. Le Corbusier en témoigne : "Le tracé régulateur est un moyen, il n'est pas une recette." (Le Corbusier, Vers une architecture, 1923). Dans cette citation, il affirme que le tracé régulateur est un outil, dans le sens où il permet - et uniquement permet - d'atteindre un résultat meilleur : il ne garantit jamais la qualité améliorée d'un résultat, car celle-ci dépend surtout de la bonne volonté de son utilisateur. De surcroît, Norman Foster illustre bien cette qualité de moyen en déclarant que "le crayon et l'ordinateur sont, s'ils sont livrés à eux-mêmes, aussi bêtes et aussi efficaces que ceux qui les conduisent." (Norman Foster, Buildings and projects of Foster Associates Volume 2, 1971-1978, 1989) et il en est de même pour le moyen du tracé régulateur.

\footnotetext{
${ }^{8}$ Le monocorde est un instrument musical à une seule corde, qui a été inventé par Pythagore pour étudier l'influence des proportions sur les sons. Quand il joue la corde à vide, puis en la pinçant à la moitié, il obtient un son qui s'accorde avec le premier, qu'on a nommé "l'octave". Quand il joue la corde à vide, puis en la pinçant au tiers, il obtient un autre son harmonieux avec le premier, qui a été appelé "la quinte". En pinçant au quart, il obtient la quarte. L'octave, la quinte, la quarte... ont alors montré à Pythagore que le proportionnement du monocorde crée des sons qui vont bien ensemble, des sons musicaux. On peut donc avancer que les proportions créent cette musicalité. Or le tracé régulateur produit ces mêmes proportions musicales, mais au lieu d'être appliquées à une corde, elles sont appliquées au dessin/morphologie d'architecture.
} 
"Son choix et ses modalités font partie intégrante de la création architecturale." (Le Corbusier, Vers une architecture, 1923). Ici, le Corbusier atteste que les modalités du tracé régulateur dépendent de la créativité de l'architecte, idée qui rejoint la qualité d'outil précédemment décrite : comme tout instrument musical, le tracé régulateur ne dicte jamais la direction d'un résultat ni d'un produit. D'ailleurs, l'auteur est libre dans sa sélection de tracés géométriques, puisqu'ils sont une mathématique absolue et infinie. C'est pourquoi leur choix dépendra du goût artistique de leur utilisateur, conjointement avec les solutions rationnelles imposées par la biologie et les contraintes du projet. Enfin, les proportions sont cette relation entre tracé régulateur, dessin d'architecture, et musique : elles produisent à travers ces disciplines la qualité d'accord.

\section{LA MYSTIFICATION DU TRACÉ RÉGULATEUR}

Car il est visuellement plaisant, le tracé régulateur obsède parfois ses utilisateurs. À cause de sa qualité de géométrie parfaite, ceux-ci se perdent dans la satisfaction du tracé géométrique : on fait gratuitement des tracés pour le fait de faire des tracés. Malgré tout, ce phénomène fait partie de l'exploration de l'outil géométrique, et permet la familiarisation avec son potentiel infini. Tout au plus, si on ne comprend pas son intérêt concret (géométrie sous-jacente pour la maîtrise logique et la musicalité visuelle de la morphologie architecturale), l'erreur de faire des tracés pour faire des tracés se perpétuera et éternisera la mystification. Sans doute, cela se produit à cause de la faible accessibilité des informations sur ce sujet millénaire. Dans la communauté des architectes, cet ésotérisme - d'intensité variable - produit deux postures extrêmes : celle des croyants aveugles, et celle des athées insensibles. D'une part, les croyants aveugles sentent la musicalité graphique, mais ne la maîtrisent pas logiquement. Dans ce pôle des intuitifs, on perçoit instinctivement la cohésion visuelle, mais la compréhension logique et intelligible reste faible : ils sont hypnotisés par la symphonie géométrique. De plus, comme les tracés régulateurs sont souvent associés aux édifices des religions dominantes (synagogues juives, églises chrétiennes, mosquées musulmanes...), le phénomène de croyance sans compréhension logique est renforcé par défaut. Ici, cette dernière s'efface au profit de l'intuition. D'autre part, les athées insensibles sont logiques, mais imperméables à la musicalité graphique des tracés régulateurs. Dans ce pôle des logiques, c'est le complémentaire de l'intuition qui se produit : n'ayant pas de maîtrise intellectuelle solide, on nie diamétralement l'intérêt des tracés régulateurs. À cet extrême du spectre, la sensibilité instinctive s'efface au profit de la rationalité. On notera que ces postures sont identiques à celles du débat sur les religions démocratisées. Sommes toutes, l'idéal serait de sentir la musicalité des tracés régulateurs, mais aussi de les comprendre intellectuellement avec raison, pour une maîtrise organique de ce sujet millénaire. Pour ce faire, le moyen de l'animation est idéal pour l'explication logique, mais aussi intuitive : non seulement, elle habilite à démystifier l'application pratique des tracés régulateurs, en montrant clairement chaque étape de leur construction. Mais aussi, elle permet de donner une saveur sonore des harmonies visuelles, ce qui aide à décrire le sentiment qu'elles produisent : on passe d'une explication mono-sens (la vue) statique et pauvre, à une explication double-sens (vue + ouïe) dynamique et riche, d'où le côté plus sensuel, plus intuitif, plus expérientiel. Exemple d'animation

\section{L'IMPACT SOCIÉTAL DU TRACÉ RÉGULATEUR}

Le musicien a des gammes pour composer, mais pas l'architecte. La gamme est la première leçon inculquée par les écoles de musique : do-ré-mi-fa-sol-la-si-do (gamme do majeur, ler cours de solfège pour enfants). L'architecte humble - par opposition au star-chitecte ${ }^{9}$ - ne connaît pas de gammes visuelles et compose dans le vide : on lui a fait croire que l'instinct suffit pour faire du grand art.

\footnotetext{
9 Un star-chitecte est un architecte de renommée mondiale, disposant de grands moyens financiers, et sollicité majoritairement pour des projets "carte-postale" à l'échelle d'un pays. Les star-chitectes ont généralement une manière de faire distincte : Zaha Hadid et Frank Gehry sont connus pour leurs projets aux formes nouvelles. En apparence, ils n'ont pas besoin du tracé régulateur pour se satisfaire de ces formes, car il semble que c'est l'algorithme de leurs logiciels qui les géométrise automatiquement. Contrairement à ces star-chitectes, l'humble-chitecte a besoin du tracé régulateur pour se satisfaire de son projet, parce que ses moyens modestes lui imposent des formes rectangulaires économiques, et à la bonne heure, le tracé régulateur permet de s'en satisfaire généreusement. De plus, il paraît que les algorithmes régulant les rectangles basaux n'existent pas encore, et après tout, il n'y en a pas besoin, car le tracé régulateur est un moyen basal largement approprié aux formes basales. Il suffit que l'architecte le cultive pour qu'il puisse se satisfaire de morphologies rectangulaires économiques.
} 
Certes, une fois sur dix, il tape accidentellement dans les bonnes cordes du tableau, et attribue ce hasard au génie de l'intuition. Il n'a pas tort : notre instinct animal est capable de sentir les harmonies visuelles, mais sa probabilité de réussite reste aléatoire. En plus de l'instinct, pourquoi ne pas bénéficier d'une certitude supplémentaire à travers une approche raisonnée ? Ceci constitue exactement l'intérêt des tracés régulateurs, car ils donnent un accès informé - non accidentel - aux gammes visuelles, aux sons accordés des yeux : désormais, l'intuition sera complétée par l'information intellectuelle, d'où les chances de réussite beaucoup plus grandes.

En outre, en France et au Maroc apparemment (peut-être dans tous les pays d'influence française), le travail de l'architecte humble restera victime du flou artistique, car on ne lui a pas enseigné le tracé régulateur. Sans doute possible, l'absence d'outils clairs - comme ce dernier - détériore sa santé en l'empêchant de dormir la nuit, étant donné que son processus de production reste vague, ce qui trouble naturellement le fleuve de la conscience, le tout couronné d'une productivité largement réduite, sans oublier une certaine tendance à la dépression. En comparaison, le travail de l'ingénieur jouit d'une netteté absolument scientifique, et cela permet à sa conscience de rester claire, à son corps de se reposer, et à son esprit d'être enthousiaste. Pendant ce temps, l'architecte s'autodétruit avec ses charrettes ${ }^{10}$ peu productives, relique retardataire dont il est victime (ce qui semble être valable en France et au Maroc, peut-être aussi dans tous les pays d'influence française). D'où la nécessité urgente du tracé régulateur, car sa nature logique chasse le flou artistique énervant, au profit d'une maîtrise rationnelle solide : l'architecte saura démontrer la mise en forme de sa conception à n'importe quel individu, au même titre qu'un

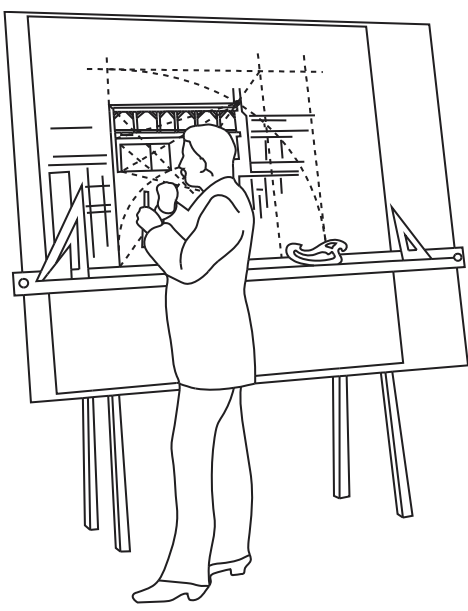

Fig. 12 : Avec le tracé régulateur, l'architecte n'est plus seulement un concepteur, il devient un concepteur musical (Driss TAOUMI 2019) mathématicien démontrant son équation. Ce faisant, il va pouvoir dompter son dessin/morphologie architecturale de manière scientifique et absolue, ce qui le mettra en confiance, et la qualité de son architecture s'améliorera naturellement, tout comme sa santé mentale et physique.

Par ailleurs, avec cet outil transcendantal, l'architecte humble répondra aux appétits sensibles de l'esprit, sans avoir besoin de ressources matérielles extravagantes. Il retrouvera alors l'un de ses arts essentiels qui le différencie de l'ingénieur ou du promoteur. Mais pourquoi cette insistance sur l'architecte humble ? Contrairement aux star-chitectes dont la grandeur des travaux semble être due aux moyens financiers, l'humble-chitecte pourra faire de grandes choses avec peu de moyens (architecture rectangulaire économique) grâce au tracé régulateur : celui-ci l'aidera à être fier de son travail, car il rend apte à se satisfaire abondamment de morphologies rectangulaires basales, lui dissipant le besoin d'effectuer des gestes formels hors du commun pour se faire remarquer : il ne subira plus de pression lui imposant de paraître extraordinaire afin que la société l'accepte. Et la mise en valeur du petit architecte est une nécessité d'autant plus urgente, car c'est lui qui conçoit les modestes constructions où on passe la majorité de nos vies (contrairement à celles des star-chitectes qu'on ne pratique que ponctuellement). Somme toutes, de l'antiquité jusqu'à maintenant, le tracé régulateur est l'un des petits plus qui ont différencié l'architecte des autres concepteurs de bâtiments. En conséquence, il lui rendra sa digne place dans la société, non seulement celle d'un concepteur de bâtiments, mais celle d'un concepteur musical de bâtiments.

\footnotetext{
${ }^{10}$ Chez les architectes d'influence française, "être charrette" signifie subir une masse de travail disproportionnée, à tel point qu'elle justifie la détérioration préméditée de sa propre santé, ceci par le recours aux nuits blanches. D’ailleurs, l'architecte est tellement habitué à en faire, qu'il a fini par donner un nom à ce masochisme architectural culte : être charrette. En effet, la pratique de l'architecte s'avère tellement floue, qu'il a besoin de sacrifier sa santé afin de boucler les travaux relatifs à ses projets (généralement, on ne boucle pas malgré les nuits blanches). Entre autres, la charrette est un concept que l'architecte subit dès qu'il s'inscrit en école d'architecture, et qu'il emporte avec lui pour le restant de sa carrière, empoisonnant ainsi sa vie professionnelle comme sa vie personnelle. Cela semble être valable dans le contexte d'influence française.
} 


\section{LA QUALITÉ SYSTÉMIQUE DU TRACÉ RÉGULATEUR}

Le tracé régulateur produit des proportions qui unifient le dessin, et l'œil semble être fait pour les lire. Contrairement aux télémètres laser, notre organe visuel est un mauvais mesureur de dimensions individuelles. Il apprécie plutôt les relations entre ces dimensions, de ce qui les rend ensemble, de ce qui les transforme en système ${ }^{11}$ : la relation proportionnelle. En ce sens, le tracé régulateur est essentiellement systémique, car il permet de travailler des rapports (de proportion). Par ailleurs, le Corbusier renchérit sur l'importance du système dans le contexte du travail humain : "Il n'y a pas d'œuvre d'art sans système." (Le Corbusier et Amédée Ozenfant, L'esprit nouveau, 1920). Clairement, il énonce que la systémique est nécessaire à toute œuvre se prétendant artistique. En effet, il semble qu'un travail sans interrelations unificatrices est perçu comme déplaisant, car il apparaît disparate ; il sonne faux, et conséquemment ne peut jamais être considéré meilleure activité de son ère - ou art -. Le Corbusier appelle alors à une qualité intégratrice et organique de tout travail humain. En architecture en tout cas, son postulat semble fondé, car beaucoup d'édifices mémorables ont été travaillés à l'aide de tracés régulateurs, qui ont permis l'ordonnancement systémique de leurs morphologies. Mais encore, peut-être que cette qualité de système est aussi responsable de leur mémorabilité historique.

En outre, le tracé régulateur crée des relations proportionnelles statiques, qui sont appliquées au dessin/morphologie d'une architecture, la transformant alors en un système statique ${ }^{12}$, soit une structure organique : si la dimension et/ou la position d'un trait/partie change, alors les dimensions et positions des autres traits/parties sont aussi altérées. Cette interrelation dimensionnelle et topologique constitue l'aspect systémique du tracé régulateur, qui de surcroît répond aux qualités suivantes : Totalité ou holisme (il permet d'unifier le dessin d'une morphologie), Dépendance environnementale (si on considère la morphologie d'une architecture comme un environnement, le tracé régulateur met en relation ses parties entre elles, grâce aux proportions. Il crée donc une dépendance environnementale entre les parties de cette morphologie), Équi-finalité (car il est une construction mathématique absolue, le tracé régulateur présente plusieurs moyens d'arriver à un même but), Causalité circulaire (voir figure 13 ci-dessous)

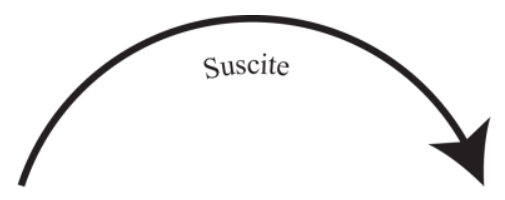

A : Tracés régulateurs

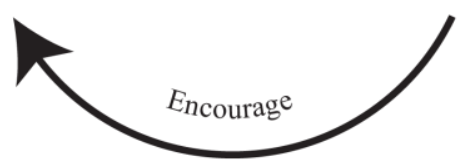

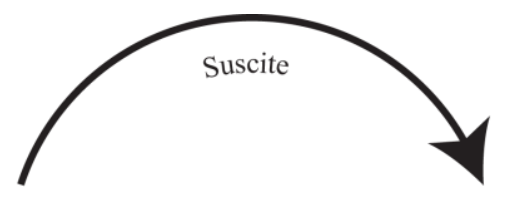

A : Tracés
régulateurs

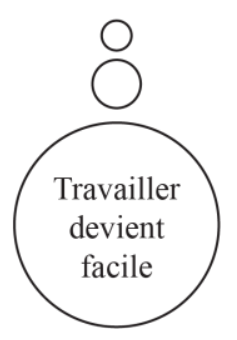

Figure 13 : Causalités circulaires comportementales des tracés régulateurs (Driss TAOUMI 2019)

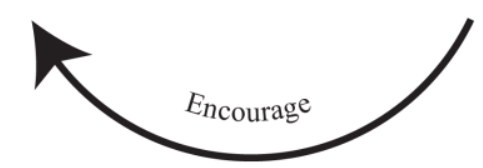

B : Le dessin devient plaisant

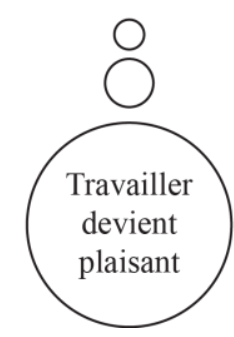

${ }^{11}$ En grec ancien, "système" vient du verbe sunistēmi, qui signifie "placer ensemble" selon Manuel lexique philologique, didactique et polytechnique ; ou, Dictionnaire portatif des sciences et des arts ... selon l'Académie française

${ }^{12}$ La définition d'un système est : "un ensemble d'éléments en interaction dynamique orientés en fonction d'un but" (Joël de Rosnay : Le macroscope, 1975). Or on remarque que le tracé régulateur introduit plutôt une interaction statique, comme il est employé sur un dessin/morphologie architecturale à priori immobile. Il est donc structure plutôt que système, à cause de sa staticité. On peut alors avancer que : "le tracé régulateur transforme un dessin/morphologie architecturale en un ensemble d'éléments en interaction statique orientés en fonction de deux buts organiques : la maîtrise constructive et l'unité visuelle" 


\section{BIBLIOGRAPHIE}

ALBERTI, Leon Battista (1452). De re aedificatoria. Florence

BLONDEL, François (1675). Cours d'architecture. Paris

BOOTWALA, Aziz V (1988). Study of geometric proportions. Kansas State University

BROADBENT, Geoffrey (1971). Metodologia del diseño arquitectónico. Barcelona : Gustavo Gili

BROADBENT, Geoffrey (1988). Design in architecture. London: David Fulton

CHAMBERS, Sir William (1759). A treatise on civil architecture. London

CHOISY, Auguste (1899). Histoire de l'architecture. Paris : Gauthier-Villars

D. K. CHING, Francis (2007). Architecture: form, space and order. John Wiley \& Sons

DE L'ORME, Philibert (1567). Le premier tome de l'architecture. Paris

ELAM, Kimberly (2011). Geometry of design. Princeton Architectural Press

EUCLIDE (-300 av. J-C). The elements of Euclid. London: William Pickering

FOCILLON, Henri (2004). La vie des formes. PUF

GALILÉE (1623). L'essayeur. Rome

GHYKA, Matila (1927). The geometry of art and life. New York: Dover Publications

GHYKA, Matila (1976). Le nombre d'or : rites et rythmes... Gallimard

GROMORT, Georges (1996). Essai sur la théorie de l'architecture. C.H. Massin

GROPIUS, Walter (1925). The new architecture and the Bauhaus. The MIT Press

GROSJEAN, J-P (1993). Le nombre d'or. H. Vial

HAMBIDGE, Jay (1926). Elements of dynamic symmetry. New York: Dover Publications

JACOBS, Michel (1926). The art of composition. New York: Doubleday

JOUVEN, Georges (1969). L'architecture cachée : tracés harmoniques. Paris : Dervy

JOUVEN, Georges (1985). La forme initiale. Paris : Dervy

KRIER, Rob (1988). Architectural composition. London: Academy Editions

LAUGIER, Marc-Antoine (1753). Essai et observations sur l'architecture. Paris

LAWLOR, Robert (1982). Sacred geometry. London: Thames \& Hudson

LE CORBUSIER (1920). L'esprit nouveau. Université du Michigan

LE CORBUSIER (1923). Vers une architecture. Paris : Crès et Cie

LE CORBUSIER (1955). Le modulor. Birkhäuser

LUND, Fredrik Makody (1921). Ad quadratum. London: Batsford Ltd

MOESSEL, Ernst (1926). Die proportionen in antike und mittelalter. Munich

MORRIS, Robert (1734). Lectures on architecture. J. Brindley

NÉROMAN, Dom (1981). Le nombre d'or - clé du monde vivant. Paris : Dervy

NEUFERT, Ernst (2014). Les éléments des projets de construction. Dunod, Le Moniteur

PALLADIO, Andrea (1570). I quattro libri dell'architettura. Venise

PERRAULT, Claude (1683). Ordonnance des cinq espèces de colonnes. Paris

REYNOLDS, Mark A. (2010). Workbooks. markareynolds.com

SERLIO, Sebastiano (1545). Livres I à IV. Venise : Giovanni Battista \& Melchiorre Sessa

STUART / REVETT (1762). The antiquities of Athens. London: J. Haberkorn

TAOUMI, Driss (2018). Les tracés régulateurs. École d'Architecture de Casablanca

VALÉRY, Paul (1921). Eupalinos ou l'architecte. Paris : Éditions de la nouvelle revue française

VITRUVE (-15 av. J-C). De architectura. Rome

VON MEISS, Pierre (1998). De la forme au lieu. Lausanne : PPUR 